\title{
Agricultura, territorio y multifuncionalidad. La experiencia de los Contratos Territoriales de Explotación
}

\author{
Eduardo Moyano Estrada' y Ana Ma Velasco Arranz ${ }^{2}$
}

Palabras clave: Sociedad rural, políticas agrarias, medio ambiente, Francia.

Key words: Rural society, Farming policies, Environment, France.

\section{Introducción}

Hasta mediados de los años 80 del pasado siglo XX, las relaciones entre el sector agrario "representado por las organizaciones profesionales y cooperativas, apoyadas por los ministerios de agricultura" y la sociedad europea "encarnada en una ciudadanía que contribuía con sus impuestos a la financiación de las políticas públicas" se basaban en una especie de pacto sociopolítico, sustentado ya en el Tratado de Roma (1958), y cuyo objetivo fundamental era producir alimentos en cantidad suficiente y a precios asequibles para la población de la naciente Comunidad Económica Europea. Durante casi tres décadas ese pacto propició un espectacular proceso de modernización de la agricultura europea, basado, sobre todo, en la mecanización de las explotaciones y en la extensión del complejo agroquímico, permitiendo alcanzar elevados incrementos de productividad y altos rendimientos agrícolas y ganaderos, como jamás se había visto antes en el sector

\footnotetext{
' IESA- CSIC. Córdoba emoyano@iesaa.csic.es

${ }^{2}$ Universidad Politécnica de Madrid a.velasco@madrid.org
} 
agrario. Ese modelo de desarrollo funcionaba a velocidad de crucero, impulsado, de un lado, por la eficacia productiva que le servía de guía y, de otro, por una Política Agraria Común (PAC) cuyo sistema de precios garantizados y protección arancelaria actuaba de malla de seguridad en muchos sectores, evitando que los agricultores tuvieran que preocuparse por la competencia exterior ni ocuparse de las externalidades negativas que iba generando su actividad "sea en forma de excedentes, sea mediante sus efectos sobre el medio ambiente.

Las primeras evidencias de las distorsiones del mercado generadas por la PAC, así como las constantes denuncias de los movimientos ecologistas sobre los modelos intensivos de agricultura, provocaron signos de preocupación en los foros agrarios allá por mediados de los años 80 . Tanto el sindicalismo agrario y el cooperativismo, como los ministerios de agricultura veían cuestionada por primera vez la legitimidad social del modelo agrícola europeo. Su idílica imagen de sistema eficiente para garantizar las rentas agrarias y asegurar el abastecimiento de alimentos y de ser una actividad integrada de forma armoniosa en la naturaleza, se deterioraba conforme surgieron problemas serios de excedentes en algunos sectores (como cereales o leche), aparecieron epidemias graves causadas por el tipo de alimentación animal (como la enfermedad de las "vacas locas") o se detectaron niveles elevados de contaminación por nitratos en las aguas subterráneas. Todo ello agravado a medida que se hacían evidentes los efectos negativos sobre los mercados mundiales del sistema proteccionista impulsado por la PAC especialmente, a partir de la inclusión de los temas agrícolas en la agenda del GATT y la OMC en lo relativo a la liberalización del comercio internacional.

En los países nórdicos y centro-europeos (Alemania y Reino Unido, sobre todo, pero también Holanda, Suecia y Dinamarca), sus políticas agrarias nacionales se verían impregnadas de lo que al gunos autores (Buttel, 1993) denominaron greening process, indicando con ese término la introducción de restricciones ambientales y de criterios orientados hacia modelos de agricultura sostenible, es decir, más respetuosa con el medio ambiente. Algunos de esos países serían pioneros en la adopción de las primeras medidas de política agroambiental cuyos efectos se harían notar más tarde a nivel europeo. Así, en 1985, se aprueba ya en el Reino Unido un programa dirigido a la conservación de algunas zonas sensibles desde un punto de vista medioambiental (zonas ESA) (Environmental Sensitive Areas), iniciativa que sería presentada al año siguiente a la Comisión Europea y que daría como resultado su inclusión como art. 19 en el Reglamento CEE 797/85, constituyendo un hito importante en la dimensión territorial de una PAC hasta entonces centrada casi exclusivamente en la regulación de los mercados. La inclusión del mencionado artículo fue acompañada de un amplio debate en el seno de la UE en torno a 
las medidas más apropiadas para frenar los efectos negativos de la agricultura intensiva sobre el medio ambiente. Ese debate giraba en torno a dos posiciones: de un lado, la posición británica, cuya propuesta ya mencionada sobre zonas sensibles (ESA) se orientaba, sobre todo, a proteger ciertos espacios naturales por su contribución a la biodiversidad, y de otro, la posición de los países mediterráneos, cuyos gobiernos apostaban por encontrar un equilibro entre el desarrollo de los modelos intensivos de agricultura "necesarios todavía en muchas zonas para reducir su déficit de modernización respecto a los países centroeuropeos" y el desarrollo sostenible de los territorios rurales "enfatizando más la dimensión económica y social de la sostenibilidad, que la dimensión ambiental" (Buller, 1996; Whitby, 1997; Alphandery, 2001; Garrido-Fernández, 2000).

A partir del citado Reg. 797/85 y su posterior desarrollo en los años 90 , se irían poniendo las bases de la política agroambiental europea, impulsada por la influencia de una opinión pública cada vez más impregnada de los nuevos valores postmaterialistas "centrados en temas relacionados con la calidad de vida y la protección del medio ambiente" y más convencida de su derecho a participar en los debates sobre la agricultura y los espacios rurales, cuestionando el monopolio ejercido hasta entonces por los grupos de intereses agrarios (Garrido-Fernández, 2006).

En ese nuevo escenario comienza a plantearse, tanto a nivel europeo como en algunos países de la UE, la necesidad de construir un nuevo sistema de relaciones entre la agricultura y la sociedad sobre bases y compromisos diferentes a los que habían guiado el proceso modernizador de los años 60 . Se abría así paso la idea de que las ayudas a los agricultores deberían estar condicionadas por el compromiso de reorientar la lógica productivista dominante en sus explotaciones e impulsar un modelo más sostenible desde el punto de vista ambiental (menos intensivo y más racional en la utilización de los recursos naturales) proponiéndose para su implementación la fórmula contractual ya probada con éxito en el Reino Unido durante la aplicación del citado art. 19 sobre zonas sensibles (ESA).

La reforma McSharry de la PAC de 1992 incorpora este tipo de compromisos en lo que serían las primeras medidas del programa agroambiental, utilizando la fórmula del contrato para su puesta en marcha. Eran medidas que permitían la concesión de ayudas a los agricultores a cambio de una serie de compromisos tales como contribuir a la conservación de zonas de especial importancia para la biodiversidad, la protección de razas ganaderas en peligro de extinción, la promoción de la agricultura ecológica o la preservación del paisaje. Las sucesivas reformas de la PAC irían en la línea de avanzar en esos nuevos compromisos, 
ampliando su contenido y extendiendo su alcance a más zonas y a mayor número de agricultores.

Tras el Consejo Europeo de Berlín de 1999 (donde se aprueba la Agenda 2000), se consagra el principio de la multifuncionalidad como eje orientador de la PAC y se institucionalizan dos pilares de la política agraria europea: un primer pilar para regular los mercados a través de las OCM e impulsar la producción agraria con fines claramente competitivos; y un segundo pilar (denominado confusamente de desarrollo rura| ${ }^{3}$ ) para promover mejoras en el uso de los factores de producción en las explotaciones, favorecer la instalación de jóvenes, facilitar el relevo generacional e impulsar el desarrollo de un modelo agrícola y ganadero más sostenible mediante la extensificación productiva y el uso controlado de los insumos agrícolas, incorporando la figura del "contrato".

Aunque la fórmula contractual estaba inicialmente circunscrita a las medidas agroambientales, su potencial innovador acabaría impregnando el debate sobre el segundo pilar de la PAC incorporando en muchas de ellas la perspectiva territorial. Algunos países, como Francia, aprovecharon esta fórmula para poner en marcha una visión amplia e integral de las relaciones entre agricultura, territorio y multifuncionalidad (dando lugar a los llamados "contratos territoriales de explotación"), mientras que otros, como España, se limitaron a continuar aplicando la vía contractual sólo en los ámbitos más restringidos del programa agroambiental (Paniagua, 2001, Garrido-Fernández, 2000; Ramos et al., 2001; Cantó, 2004).

El objetivo de este artículo es analizar la experiencia de los "contratos territoriales de explotación" (en adelante CTE) en Francia, estudiando el contexto político y social en el que surgen y destacando su potencialidad como vía para hacer realidad una nueva forma de entender las relaciones entre los agricultores y la sociedad "un nuevo pacto social, por utilizar una expresión tan querida por los analistas y políticos franceses". Para ello partimos de la tesis de que los CTE eran un instrumento político que ofrecía la posibilidad de integrar la dimensión territorial en la agricultura y de recuperar la dimensión agraria (renovada y multifuncional) en las estrategias de desarrollo rural. Sin embargo, su aplicación estuvo rodeada de un ambiente político-sindical tan agitado y convulso, que acabó contaminando el debate sobre los CTE, a los que se les juzgó más por ser un producto de la política socialista de Lionel Jospin, que por su capacidad para introducir cambios en la política agraria

\footnotetext{
${ }^{3}$ En realidad, el segundo pilar se basaba en una reactivación de la tradicional política de estructuras agrarias, con la novedad de incluir el programa agroambiental que antes estaba regulado por un reglamento específico.
} 
francesa. La tensión política fue tan elevada, que los CTE no pudieron resistir el cambio producido en 2002 en Francia con la victoria de Chirac en las elecciones presidenciales y la posterior formación de un gobierno conservador, siendo reformados y convertidos en los CAD (contratos de agricultura durable-sostenible), de características muy similares, hasta su plena desaparición.

Aunque de corto recorrido (apenas tres años de vigencia), la importancia de los CTE trasciende el ámbito de la agricultura francesa, por cuanto que, en el actual escenario de aplicación del último reglamento de desarrollo rural (Reg. $1.698 / 2005$ ) financiado por el nuevo fondo europeo FEADER, estas fórmulas contractuales están siendo utilizadas hoy como referencia en algunos países de la UE (como Reino Unido, Suecia, Países Bajos y España), habiéndose incluido en sus correspondientes PNDR (Planes Nacionales de Desarrollo Rural) para el periodo 2007-2013. Concretamente, en el PNDR español se incluyen los "contratos territoriales de explotación" como opción facultativa para aplicar los Ejes 1 y 2 del Reglamento por parte de los gobiernos regionales. Asimismo, y continuando con el caso español, la huella de los CTE puede verse también en el actual proyecto de Ley de Desarrollo Sostenible del Medio Rural, donde se establece la figura de los "contratos territoriales de zonas rurales". Todo ello justifica el interés de abordar el estudio de los CTE, aunque sólo sea con el propósito de mostrar cómo se implementó una modalidad de contrato cuyo objetivo era innovar en el ámbito de la política agraria, superando la visión sectorial e introduciendo una perspectiva más amplia e integral en la que se valoraban las implicaciones de la actividad agrícola y ganadera en el territorio circundante.

\section{Contexto, naturaleza y objetivos de los CTE}

Los CTE eran un instrumento de política agraria creado por la Ley de Orientación Agraria (LOA) francesa de 9 de julio de 1999. El objetivo de los CTE se enmarcaba en los objetivos más generales de esa Ley, a saber: incentivar a los agricultores para adaptar la gestión de sus explotaciones a las exigencias de un nuevo escenario marcado por el principio de la multifuncionalidad. En este sentido puede decirse que, como en tantas otras ocasiones, el gobierno francés (sea cual fuere su color político) se anticipaba a las decisiones europeas, concretamente a la Agenda 2000 aprobada ese mismo año en el Consejo Europeo de Berlín, donde, como se ha señalado, se consagraba la multifuncionalidad de la agricultura como principio rector del segundo pilar de la política agraria europea. Los CTE nacen, por tanto, en paralelo a la Agenda 2000 y en concomitancia con el 
primer Reglamento de desarrollo rural (Reg. 1.257/1999) surgido del escenario presupuestario 2000-2006.

Con los CTE, el agricultor signatario del contrato se comprometía a cumplir una serie de compromisos relacionados no sólo con la dimensión productiva de su explotación (tipos de cultivos, utilización de insumos, tratamientos fitosanitarios, formas de gestión,...), sino también con su dimensión ecológica (preservación de los recursos naturales del territorio circundante; bienestar y salubridad de los animales; aprovechamiento de los recursos hídricos...) y cultural (valorización del patrimonio cultural, conservación de los recursos arquitectónicos ligados a la explotación,...), así como con su dimensión socioeconómica (creación y/o mantenimiento de empleo, mejora de las condiciones laborales,....). Los CTE eran concebidos como un instrumento de desarrollo a través del cual se le daba oportunidad al agricultor para que integrara su explotación agraria (y el patrimonio cultural y natural ligado a ella) en una estrategia territorial de desarrollo rural y agrario. Con los CTE, el gobierno de Jospin transformaba el clásico debate técnico sobre la modernización de la agricultura en un debate social, convirtiendo los temas agrarios en asuntos de interés general y dando voz a grupos de intereses no relacionados directamente con la agricultura.

El giro que le dio el gobierno socialista francés al debate sobre la agricultura se explicaba por varias razones. En primer lugar, por el debate, muy presente entonces en Francia, sobre la legitimidad social de las ayudas agrícolas y sus efectos sobre la distribución de las rentas y la dinamización de los territorios rurales. Desde los años 60, la politica agraria francesa se había transformado en una politica fuertemente elitista, guiada por criterios productivistas y basada en una concentración de las ayudas y los medios de producción en un cada vez más reducido grupo de agricultores. Ese modelo selectivo de modernización agrícola, apoyado durante más de treinta años desde los poderes públicos, como ruta a seguir por los agricultores, había conducido a una fuerte reducción de la población agraria en los campos franceses, además de provocar efectos nocivos en la salud de los consumidores y de contaminar el medio ambiente. Junto a eso, y quizá como uno de los principales argumentos esgrimidos por sus detractores, ese modelo intensivo y especializado de agricultura era muy costoso económicamente, perdiendo sentido y justificación en un escenario de excedentes y apertura de los mercados mundiales. En definitiva, a principios de los años 90 había sobre la mesa del debate social y político en Francia un conjunto de buenas razones para cuestionar el modelo de agricultura que había dominado el panorama francés y europeo de las tres últimas décadas, pero sin tener otro modelo alternativo que lo reemplazase (Remy, 2004). 
En segundo lugar, había otro tema que estaba presente en el debate social sobre la agricultura y que venía de lejos, justo casi desde comienzos de los años 70, atravesando los círculos del poderoso sindicalismo agrario francés. Nos referimos al tema de la pluriactividad y sus efectos, positivos o negativos según se mire (radicando ahí precisamente la base de la controversia), en el desarrollo de la agricultura y el mundo rural. En torno a ese tema se venían planteando dos posiciones de claro contenido político e ideológico. Por un lado, una posición calificada de "profesional", defendida por la plana mayor del sindicalismo mayoritario "representado por la FNSEA (Féderation Nationale des Syndicats des Exploitants Agricoles) y el CNJA (Centre Nacional des Jeunes Agriculteurs)" y corroborada entonces a nivel europeo por el COPA (Comité de Organizaciones Profesionales Agrarias), según la cual la pluriactividad tenía efectos perniciosos para el desarrollo agrícola y, por ello, debía ser combatida con programas que facilitaran la instalación a tiempo completo de jóvenes agricultores en explotaciones modernas y viables. Por otro lado, se encontraba una posición calificada de "campesina" (paysanne), defendida por sindicatos como la CNP (Confédération Nationale Paysanne) y el MODEF (Mouvement pour la Defénse de l'Explotation Familielle), que venían apostando por el reconocimiento de la pluriactividad como vía para impedir el abandono de muchos pequeños agricultores al darles la oportunidad de seguir en su explotación mediante la diversificación de sus rentas, contribuyendo con ello a fijar población en el medio rural. Al apoyo de la pluriactividad se le añadía el argumento de que los llamados despectivamente "agricultores pluriactivos" eran los que menos ayudas públicas recibían y los que lograban mantener la viabilidad de sus explotaciones gracias a la renta obtenida con actividades realizadas fuera de la agricultura contribuyendo al desarrollo de determinadas zonas rurales (Bazin y Roux, 1995).

En tercer lugar, la aprobación en 1992 de la mencionada reforma McSharry de la PAC será otro elemento que animará el debate social sobre la agricultura, al introducir cambios fundamentales en la distribución de las ayudas agrícolas y abrir nuevas líneas programáticas para innovar en materia de política agraria. Nos referimos por supuesto a la eliminación del sistema de precios de garantía y a su sustitución por un sistema de pagos por hectárea o cabeza de ganado, pero, sobre todo, nos referimos a las llamadas "medidas de acompañamiento" "calificadas así por acompañar a las nuevas medidas de regulación de mercados dirigidas a la reducción de excedentes", entre las que destacaba el programa de reforestación de tierras agrícolas, la retirada de tierras (set-aside), el cese anticipado de la actividad (jubilación anticipada) y el programa agroambiental. Este cambio suponía un giro copernicano en la filosofía de la PAC, puesto que hasta ese momento al agricultor se le había venido remunerando por producir, y cuanto 
más mejor. Además, abría el debate en torno a qué tipo de agricultor debía ser el destinatario de las ayudas públicas, lo que equivalía a discutir sobre quién era merecedor de quedarse en la profesión agrícola $y$, en definitiva, sobre qué modelo de agricultura debería basarse el futuro de la UE (Velasco, 2002; Grupo de Brugge-Brujas, 1997).

En cuarto lugar, la Declaración de Cork (1996) marcará las líneas del debate europeo sobre el mundo rural, ya que, en esa conferencia, celebrada en la ciudad irlandesa del mismo nombre y propiciada por la Comisión Europea (concretamente, por el entonces comisario de Agricultura, el austriaco Franz Fischler), se reconoció la diversidad de los espacios rurales y se planteó la necesidad de favorecer la diversificación de actividades en ellos con objeto de frenar el creciente despoblamiento de los territorios europeos (Delgado, 2004 y 2005). Además, en el marco de la citada conferencia, surgieron las primeras ideas para definir una política específica de desarrollo rural en la que, junto al apoyo a la iniciativa Leader, se contemplara la agricultura como un sector capaz de integrar múltiples funciones. Asimismo, de dicha conferencia se refuerza el discurso de lo "rural" como "fuente de calidad de vida para la sociedad urbana", potenciándose una imagen de la "ruralidad" en la que destacan no sólo los actores (los agricultores y sus rentas), sino también las figuras (paisaje), el patrimonio natural (medio ambiente) y cultural (territorio, folklore, ...) y las prácticas (actividades agrarias y no agrarias). El agricultor y su trabajo siguen estando presentes en ese discurso, pero ya no exclusivamente en su función de producir alimentos, sino con la misión de contribuir a mantener vivos y en buen estado de conservación los espacios naturales y los territorios.

En quinto lugar, la ya citada Agenda 2000 abrirá un nuevo escenario para la agricultura europea y francesa al reformar los fondos estructurales y orientar la PAC hacia una paulatina reducción de las ayudas del primer pilar (en respuesta a las exigencias de la $\mathrm{OMC}$ ), preparándola para afrontar la ampliación de la UE a nuevos Estados. La importancia de la Agenda 2000 radica en el hecho de que la Ley de Orientación Agraria se anticipa a ese nuevo escenario creando un instrumento político (los CTE) cuya financiación se preveía afrontar en el marco propiciado por la mencionada Agenda.

Puede decirse, en definitiva, que la creación de los CTE se enmarcaba en los debates más generales sobre la agricultura y la política agraria que se estaban desarrollando a nivel europeo, representando un avance en la definición de un nuevo modelo de desarrollo agrario basado en el principio de la multifuncionalidad, un modelo en el que la agricultura recupera tanto su dimensión territorial (relegada a un segundo plano durante la etapa de productivismo a ultranza), como social 
(incorporando entre sus fines el mantenimiento y creación del empleo) y medioambiental (incluyendo entre sus prioridades la conservación del medio ambiente) (Gómez-Limón y Barreiro, 2007). Los CTE se crean, pues, para dar cuerpo a un enfoque de intervención pública en la agricultura francesa proponiendo una gestión contractual de la actividad agraria. En este sentido cabe presentarlos como un instrumento dirigido a mentalizar a los agricultores sobre la necesidad de que las ayudas públicas a la agricultura no estén sólo orientadas a la producción, sino que sean fruto de una contrapartida por la prestación de otros servicios de interés general. La importancia del nuevo enfoque consistía en lanzar el mensaje a los agricultores de que había que producir de otra manera y de que era necesario afrontar un cambio en el modelo de producción agrícola, poniendo en marcha nuevos instrumentos políticos que fomenten estrategias de desarrollo sostenible.

A diferencia de otros gobiernos de la UE, que no crearon tales herramientas para acompañar la política de desarrollo rural, sino que se limitaron a cumplir los mínimos requisitos impuestos por la política agroambiental, el gobierno francés fue pionero en la implementación de una nueva política orientada a alcanzar un modelo de agricultura multifuncional y sostenible. Eso explica el interés suscitado por la experiencia de los CTE franceses en otros países de la UE, a la hora de impulsar el debate sobre la necesidad de introducir fórmulas contractuales similares en el momento de implementar, como se ha señalado, el nuevo Reglamento de Desarrollo Rural (FEADER).

\section{Estructura y contenido de los CTE}

Mediante los CTE se incentivaba a los agricultores para que desarrollaran un proyecto global en sus explotaciones, introduciendo cambios en el modo de gestionarlas y definiendo sus estrategias productivas dentro de una visión integral de las relaciones entre agricultura y territorio y de acuerdo con las prioridades establecidas en la nueva Ley de Orientación Agraria. El carácter global del proyecto significaba que la explotación agraria debía ser considerada como un todo, tanto desde el punto de vista social (las distintas personas que la componen, sean su titular, las ayudas familiares o la mano de obra asalariada), como estructural (capital físico, maquinaria y factores de producción), lo que venía a suponer un cambio importante respecto a la vieja política estructural orientada casi exclusivamente a financiar acciones de mejora guiadas por una lógica económica de productividad.

Desde el punto de vista de la estructura y contenido de los CTE, se partía de un diagnóstico inicial de la explotación, se marcaban unos objetivos y se preveía, tras 
los cinco años de duración del contrato, un punto de llegada respecto a factores tales como los siguientes: el nivel de renta a alcanzar, la contratación de mano de obra asalariada, el autoempleo del trabajo familiar, la instalación de jóvenes agricultores, las prácticas agrícolas y ganaderas, la utilización de insumos, el uso y explotación de los recursos naturales, la interacción de la actividad agraria con el entorno, la conservación y rehabilitación del patrimonio natural y cultural,... Para todo ello, el agricultor recibía el asesoramiento de los técnicos de la Cámara Agraria de su provincia, además del que podría solicitar de sus organizaciones profesionales o cooperativas.

Las transformaciones a realizar en la explotación se recogían en dos capítulos: uno, socioeconómico, y otro medioambiental. En el capítulo socioeconómico se describían las acciones comprometidas en el ámbito de los factores relacionados con la renta o el empleo, se cuantificaban las inversiones necesarias y se definían los objetivos a alcanzar en términos de rentabilidad económica. En ese capítulo se incluían acciones tales como las siguientes: mejora de las estructuras ganaderas (por ejemplo, rehabilitar los establos y las naves de almacenamiento); transformación de los sistemas productivos (por ejemplo, pasar de los sistemas de agricultura convencional a la producción ecológica o integrada); mejora de la calidad de las producciones (integrándolas, por ejemplo, en una denominación de origen o en una certificación de calidad); creación de empleo (ya sea contratando mano de obra asalariada o regularizando la situación laboral del cónyuge y del trabajo familiar ligado a la explotación); desarrollo de nuevas actividades (turismo rural, venta directa de los productos, artesanado,...). El máximo de ayudas fijado para financiar el capítulo socio-estructural era de 100.000 FF (unos 15.000 euros) por explotación, cifra equivalente como media al $40 \%$ de las inversiones totales previstas en el contrato.

En el segundo capítulo, denominado medioambiental, se exponían las acciones comprometidas con el objetivo de promover una interacción más respetuosa entre la actividad agraria, el medio ambiente y el entorno natural de la explotación. Entre tales cambios cabe señalar los siguientes: nuevas prácticas agrícolas y ganaderas (en la línea de extensificar las producciones y mejorar el bienestar y salubridad de los animales); utilización más racional de los insumos (fertilizantes, pesticidas y herbicidas, para mitigar los efectos contaminantes) y factores de producción (suelo y agua); limpieza de acequias y puntos de agua para evitar inundaciones; cuidado de los caminos rurales y del espacio forestal; mejora y revalorización del patrimonio natural y cultural de la explotación (rehabilitación de las casas rurales o de otras edificaciones de valor histórico o arquitectónico, reconstrucción de las vallas o muros de piedra, ...). En este segundo capítulo se cuantificaban 
las inversiones a realizar y se evaluaban los posibles sobrecostes que tales actuaciones podrían suponerle al agricultor para compensarlo económicamente "en función de lo que dejara de ganar en términos de rentabilidad económica con esos cambios". Las ayudas ligadas al capítulo medioambiental se determinaban por hectárea de superficie, tal como se venía haciendo en las medidas del programa agroambiental, y su cuantía era decreciente con la superficie declarada. Aunque formalmente no había un tope de ayudas para este capítulo, en la práctica se fijaba en un máximo de 100.000 euros por CTE. Los objetivos marcados y las inversiones previstas debían lograrse de manera conjunta, sin posibilidad alguna de que se cumpliera sólo el capítulo socioeconómico, pero no el medioambiental, o viceversa. Desde el principio, los temas relacionados con la financiación de los dos capítulos y con la posibilidad de trasladar fondos de uno a otro, dieron lugar a enconados debates entre las diferentes organizaciones agrarias, tal como veremos más adelante, mostrándose ahí las diferentes concepciones sobre el modo de gestionar la agricultura y el mundo rural.

La instrucción de los informes relativos al seguimiento y supervisión de los CTE correspondía a las Directions Départementales de l'Agriculture (DDA) (equivalentes a nuestras delegaciones provinciales de agricultura). Para que el contrato fuera válido, el agricultor se comprometía a cumplir un cahier des charges, que debía ser aprobado por la Comission Départementale d'Orientation Agricole (CDOA), comisión creada expresamente por la Ley de Orientación Agraria y formada a nivel provincial por organizaciones profesionales y cooperativas, asociaciones ecologistas, forestales y de cazadores, además de por representantes de la administración (agricultura, industria, sanidad, medio ambiente, trabajo y asuntos sociales, consumo,...). Es importante subrayar tres aspectos de dicha Comisión: en primer lugar, la pluralidad de su composición, que significaba una forma de abrir el debate sobre la agricultura a nuevos grupos sociales; en segundo lugar, la designación del territorio como espacio de negociación política sobre los temas agrarios, que implicaba un cambio significativo respecto al modo tradicional de hacer política agraria en Francia (ahora más territorial y multifuncional, y menos sectorial que antes), y en tercer lugar, la presencia en la citada Comisión de responsables de diversos departamentos de la administración pública (rompiendo el tradicional monopolio del ministerio de agricultura). 


\section{Los CTE en el marco de la política de desarrollo rural}

Como ha sido habitual en otras ocasiones, los gobiernos franceses no se han limitado a esperar instrucciones de Bruselas, ni a aplicar de forma automática los reglamentos de la UE, sino que han sabido aprovechar el margen de maniobra que se les deja para imprimir su propia impronta a la política agraria y rural. Cada periodo de reforma de la PAC ha ido, por lo general, acompañado en Francia de una ley de orientación agraria, donde el legislador ha marcado las posibilidades de avanzar en los cambios necesarios para la agricultura francesa sin tener que acompasarse al ritmo de la normativa europea (Servolin, 1989; Hervieu, 1996).

En el caso de la mencionada Agenda 2000 y la reforma emprendida en el ámbito del segundo pilar de la PAC, el gobierno francés se anticipa, introduciendo, como se ha señalado más arriba, la fórmula innovadora de los CTE. Una vez aprobado el Reg. 1.257/1999 de Desarrollo Rural (surgido precisamente del escenario presupuestario 2000-2006) integra los CTE en el Plan Nacional de Desarrollo Rural (PNDR) francés para cofinanciarlos con fondos procedentes de ese reglamento, así como con recursos derivados de aplicar en Francia la modulación de las ayudas del primer pilar de la PAC, y con recursos de las políticas de desarrollo regional.

En el PNDR francés se fijaban seis ejes prioritarios, siendo uno de ellos el correspondiente a las acciones cuya aplicación se preveía realizar mediante CTE. Casi la mitad (un 42\%) del presupuesto del PNDR 2000-2006 se destinaba a financiar las acciones previstas en los $\mathrm{CTE}$, y el resto (un 58\%) iba destinado a los otros cinco ejes, distribuyéndose del siguiente modo: reducción de los desequilibrios territoriales $(41,0 \%)$, promoción de la calidad de las producciones agrarias y aumento del valor añadido de las explotaciones (13,0\%), puesta en valor de los recursos forestales $(1,8 \%)$, protección y valorización del patrimonio ecológico $(1,8 \%)$ y organización de programas de formación dirigidos a los agricultores $(0,6 \%)$. En lo que se refiere a acciones concretas, el PNDR seleccionaba 16 medidas de las 22 incluidas en Reg. 1.257/99 de desarrollo rural, y establecía que diez de ellas se aplicarían a través de CTE, bien en el capítulo socioeconómico o en el de medio ambiente (Cuadro ${ }^{\circ} 1$ ).

Vemos así, que las inversiones en las explotaciones (planes de mejora), el programa de jubilación anticipada, las medidas del programa agroambiental, la reforestación de tierras agrícolas (y otras medidas forestales), la mejora de la comercialización, la valorización del patrimonio (natural y cultural), la diversificación de las producciones, la gestión de la calidad del agua y las acciones de protección del medio ambiente, eran implementadas a través de la fórmula contractual que representaban 
los CTE. Mediante los CTE se recuperaba la dimensión territorial de la agricultura, lo que constituía un cambio importante en la aplicación de la política de estructuras en Francia, una política centrada hasta entonces casi exclusivamente en una visión productivista de la actividad agraria.

Cuadro I.Acciones incluidas en el PNDR francés (2000-2006)

Inversiones en las explotaciones agrarias (a)

Nuevas instalaciones de jóvenes agricultores (b)

Programas de formación (c)

Jubilación anticipada (d)

Ayudas a zonas desfavorecidas y de montaña (e)

Medidas agroambientales ( $f$ )

Transformación de los productos agrarios $(\mathrm{g})$

Programa de reforestación de tierras agrícolas (h)

Otras medidas forestales (i)

Mejora de los accesos y caminos agrícolas (i)

Ordenación y concentración parcelaria $(k)$

Mejora de la comercialización agraria $(\mathrm{m})$

Valorización del patrimonio natural y cultural (o)

Diversificación de las producciones ( $p$ )

Gestión de la calidad del agua (q)

Protección del medio ambiente $(t)$

Nota: Las letras se corresponden con las 22 medidas del Reg. 1257/1999 (en negrita, las acciones canalizadas a través de los (TE). Las medidas $\mathbf{I}, \mathbf{n}, \mathbf{r}, \mathbf{s}, \mathbf{U}, \mathbf{v}$ del Reglamento europeo no están incluidas en el PNDR francés por no haber sido consideradas prioritarias por el gobierno.

Elaboración propia.

La idea de articular el segundo pilar de la PAC con el territorio no era nueva, pues ya en los años 80 se había incorporado el objetivo de la cohesión territorial en la política europea (a través de los fondos estructurales), incluyendo, por ejemplo, dentro de la política socioestructural las indemnizaciones compensatorias para los agricultores con explotaciones ubicadas en zonas desfavorecidas o de montaña. La novedad que añadían los CTE era extender la dimensión territorial de la agricultura a todas las áreas y espacios rurales y no sólo a las zonas desfavorecidas. Con los CTE se incorpora la idea de que la agricultura es una actividad que tiene 
implicaciones territoriales independientemente de cual sea la estrategia productiva del agricultor y que esas implicaciones lo mismo se reflejan en los recursos naturales (suelo y agua), que en el paisaje, el patrimonio cultural, la biodiversidad o incluso el despoblamiento o la calidad de vida en el medio rural.

Estaba presente, por tanto, en los CTE una concepción amplia de las relaciones entre agricultura y territorio, lo que suponía un avance importante respecto a la visión más restrictiva de los años 80 , abriendo un interesante debate sobre las limitaciones de las políticas agrarias y rurales que se estaban aplicando entonces en la UE. De acuerdo con ese punto de vista, estas políticas serían insuficientes para garantizar la calidad de vida en el medio rural y asegurar una articulación armoniosa y sostenible entre la actividad agraria y el entorno circundante, ya que se plantean desde una visión demasiado sectorial y restrictiva de la agricultura y el territorio. De ahí la necesidad de integrarlas en el marco de políticas de ordenación territorial gestionadas por agencias interministeriales y no por los departamentos agrarios (Velasco, 2002). Ese debate está presente hoy en muchos países de la UE, y, en el caso de Francia, está en la base del nuevo PNDR para el periodo 2007-2013, donde se plantea la necesidad de unir todos los fondos estructurales para financiar acciones de desarrollo integradas en el marco de una política amplia de ordenación del territorio.

\section{Implicaciones sociales y politicas de los CTE}

La aplicación de los CTE conllevó una serie de efectos sociales y políticos, que trascendían el ámbito de la agricultura francesa. Entre esos efectos destacan los siguientes: haber impulsado mecanismos de participación social no corporativista; haber significado una ruptura con el modelo unidimensional de agricultor profesional dándole un nuevo sentido a la pluriactividad, y haber dinamizado los servicios técnicos de las organizaciones profesionales y de la administración pública a nivel local adecuándolos a las nuevas demandas de los agricultores y a las orientaciones multifuncionales de la agricultura.

\section{I. Participación social no corporativista}

Con los CTE, el debate sobre la agricultura se abrió a nuevos actores (silvicultores, ambientalistas, redes de desarrollo rural, cazadores, ...), rompiendo, por un lado, el monopolio exclusivo de las organizaciones profesionales agrarias en el ámbito 
de la interlocución con los poderes públicos, y, por otro, reduciendo el protagonismo del Ministerio de Agricultura en la regulación del sector al incorporar en estos asuntos a otros departamentos ministeriales, como los de medio ambiente u ordenación del territorio. En este sentido puede decirse que los CTE impulsaron mecanismos de participación no corporativa favoreciendo la emergencia de una pluralidad de lógicas de actuación y la presencia de actores institucionales internos y externos al ámbito de la agricultura. Esta pluralidad se manifestaba en el seno de las ya citadas Comisiones Departamentales (provinciales) de Orientación Agraria (CDOA), que eran precisamente las encargadas de definir las estrategias de desarrollo para cada territorio, priorizando las acciones dentro de las posibilidades ofrecidas por el PNDR. Es decir, eran los representantes de la sociedad civil (y no sólo las organizaciones profesionales agrarias, como ocurría antes) los que, en el seno de las CDOA, definían las estrategias de desarrollo agrícola y rural más adecuadas para sus correspondientes territorios, marcándoles a los agricultores las acciones prioritarias a seguir en los CTE.

La naturaleza abierta y plural del procedimiento de los CTE es lo que explica que se hable de ellos como la concreción de un nuevo pacto social entre la agricultura y la sociedad, distinto de los acuerdos corporativos que antaño se hacían dentro y para el propio sector agrario. Según Jacques Remy (2001) las sesiones de debate en las CDOA eran buena prueba de la existencia de una dinámica de negociación colectiva para definir nuevas funciones y nuevos perfiles profesionales de los agricultores franceses, participando en ella grupos sociales que partían de diferentes concepciones ideológicas y de posiciones diversas sobre cómo debía articularse la relación entre agricultura, territorio y mundo rural.

\subsection{Ruptura con el perfil unidimensional del agricultor profesional}

Los CTE significaron una ruptura con las leyes de orientación agraria de los años sesenta, leyes que, como se ha señalado, definían un modelo ideal de agricultor que se basaba en una concepción exclusivamente profesional de su actividad y enfatizaba su dimensión productiva (Coulomb y Nallet, 1980). A diferencia de las anteriores, la Ley de Orientación Agraria de 1999 reconocía que los agricultores, además de producir alimentos, desarrollan otras actividades (venta de productos en la explotación, conservación de recursos forestales, ocio y recreación, preservación del paisaje, artesanía,....) que contribuyen a mantener vivos y dinámicos los espacios rurales. En consonancia con ello, se establecía que los agricultores debían aprender nuevas formas de gestionar sus explotaciones, puesto que el objetivo de su actividad no consiste sólo en lograr el máximo de productividad 
agraria. Concebida ahora como una profesión multifuncional, el agricultor habría de actuar como un actor polivalente capaz de adaptarse a la variedad de funciones que la sociedad demanda a la agricultura. Las nuevas ocupaciones de los agricultores requerirían de nuevas competencias y de una voluntad firme de abrirse al exterior para aprovechar el contexto de oportunidades que se les presenta (Remy, 2004).

Los CTE fueron, en definitiva, el instrumento instaurado por la Ley de Orientación de 1999 para permitir a los agricultores adaptarse a ese nuevo escenario multifuncional de la agricultura y responder a las demandas de la sociedad. El objetivo de los CTE era incentivar un modelo de agricultura de servicios que diversificara las rentas de los agricultores, fuera compatible con las exigencias del desarrollo sostenible, atendiera las demandas de los consumidores $y$, en definitiva, justificara la concesión de ayudas públicas. Es en este sentido en el que cabe afirmar su carácter innovador y el potencial de cambio que conllevaban.

\subsection{Dinamización de los servicios técnicos a nivel local}

La puesta en marcha de los CTE llevaba asociado un programa específico de formación dirigido a técnicos y asesores de las Cámaras Agrarias y de las organizaciones profesionales, además de otro programa de formación e información destinado a los agricultores. En este sentido puede decirse que los CTE significaron una especie de revulsivo dentro del sector agrario por cuanto que muchos técnicos de los organismos profesionales y de la propia administración agraria encontraron de nuevo la oportunidad de volver al campo y promover sobre el terreno un nuevo modelo de desarrollo agrícola y rural, recuperando así su vieja función de asesoramiento, dinamización y extensión. Al hilo de los CTE se renovaron los antiguos servicios de extensión agraria (ligados en unos casos a las Cámaras Agrarias y en otros a las organizaciones profesionales o cooperativas) cuyas funciones habían quedado reducidas a meras labores de gestión administrativa de las ayudas de la PAC. Ahora, desprendidos de su ribete agrarista, $y$ en un proceso que puede calificarse de refundación, impulsado en la etapa de los CTE, estos servicios de extensión cumplen una tarea de formación e información a los agricultores para adaptarlos al nuevo escenario de la multifuncionalidad y el desarrollo sostenible.

Estas tareas se desarrollaban, y continúan desarrollándose, sobre todo a nivel local, ya que con los CTE no se pretendía favorecer un determinado sistema de producción especializada, sino aprovechar toda la diversidad agrícola y ganadera existente en el territorio. La diversidad se convierte en un valor que debía ser aprovechado a 
través de los compromisos adquiridos por el agricultor en el correspondiente CTE. Como hemos señalado, cada CTE estaba asociado a un proyecto de explotación definido por el agricultor con la ayuda de técnicos de la Cámara Agraria lo de las organizaciones profesionales), pero integrado en una serie de prioridades socioeconómicas o medioambientales establecidas previamente en la CDOA para el conjunto del territorio (una comarca, una petite region o una provincia) ${ }^{4}$. De este modo, los CTE pueden verse como instrumentos de agregación de intereses a nivel local, ya que es en ese nivel donde se definían las estrategias para impulsar un nuevo modelo de agricultura y valorar sus implicaciones sociales y territoriales. No era una estrategia definida desde arriba, a nivel estatal o regional, sino en niveles más cercanos al territorio y como resultado del consenso entre los actores sociales e institucionales allí existentes.

\section{Resultados de los CTE}

Aunque hasta final de 2007 no se podrá conocer el verdadero impacto de los CTE "ya que ese año finalizará el primer periodo quinquenal de todos los contratos firmados", un primer balance realizado por el ministerio francés de Agricultura a finales de mayo de 2003 mostraba algunos datos interesantes 5 . Se habían firmado 46.810 contratos individuales (afectando a una superficie de 4,6 millones de hectáreas), lo que significaba que algo más del $13 \%$ de los agricultores franceses a tiempo completo se habían comprometido con esta vía contractual, recibiendo una media de 42.000 euros por contrato (con una dotación global de 2.000 millones de euros para el conjunto de los CTE).

La cantidad de contratos suscritos había sido diferente según las regiones (el 8,2\% de las explotaciones en Poitou-Charentes y el 8,1\% en Basse Normandie frente al $3,5 \%$ en la Bretagne, por ejemplo), siendo menor en los departamentos (provincias)

\footnotetext{
${ }_{4}^{4}$ Por ejemplo, en la región de Bretaña, una de las medidas que formaban parte del plan regional para reducir el problema de contaminación del agua por nitratos era incentivar la disminución de la cantidad utilizada de azote (abono líquido nitrogenado), lo que explica que se incluyera entre las acciones a promover en los CTE. En el suroeste, en la Dordogne, entre las medidas más divulgadas a través de CTE estaba la integración de la producción de pato y sus transformados (confit de canard, foie de canard, etc.) en denominaciones de origen.

${ }^{5}$ El Ministerio francés de Agricultura y Pesca y el Centre National pour l'Aménagement des Structures des Exploitations Agricoles (CNASEA) publicaron a finales de 2003 un informe con los primeros resultados.
} 
del centro y noroeste "con sistemas agrarios más orientados a la producción (sea cerealista o ganadera)", y mayor en los del sur "formados por zonas desfavorecidas o de montaña (de hecho, el 60,6\% de los contratos se firmó en estas áreas)" (ver mapa de la Figura 1). Esto corrobora la idea de que los CTE eran percibidos más como instrumentos de desarrollo rural-territorial que de modernización, lo que explica que apenas fuera utilizado por los grandes cerealistas del Bassin Parisien.

Aunque apenas un $13 \%$ de los CTE suscritos correspondían a proyectos de agricultura ecológica, en este eje es donde se habían realizado las inversiones más importantes (la media por contrato de agricultura ecológica se elevaba a 45.500 euros, frente a los 42.000 euros de media para el total de contratos). Más de la mitad de los CTE habían sido suscritos por ganaderos, centrándose sus proyectos en la mejora de la gestión extensiva de praderas; en este sector, los CTE fueron una gran oportunidad para que los ganaderos diversificaran sus producciones e integraran la producción de carne en filières de calidad permitiéndoles afrontar la crisis de la EEB (vacas locas) que por aquellos años afectaba a la ganadería francesa. Cerca del $20 \%$ de los CTE fue suscrito por agricultores de explotaciones herbáceas o de grandes cultivos (COP), siendo el objetivo de estos contratos reducir los insumos en la explotación en línea con el modelo de agricultura sostenible que se estaba impulsando en Francia. Finalmente, cabe señalar que uno de cada 10 CTE llevaba entre las actuaciones comprometidas en el contrato la promoción del turismo rural, y en la mitad se incluían acciones destinadas a la conservación del patrimonio fundiario (en forma de rehabilitación de algún edificio singular de la explotación).

En cuanto a las acciones destinadas al mantenimiento y creación de empleo, cabe señalar que, entre 2000 y 2003, por cada 15 CTE se creó un nuevo empleo en la agricultura, lo que significó la creación de 1.400 empleos agrícolas en ese periodo. La vía de los CTE fue también muy utilizada para la instalación de jóvenes agricultores, de tal modo que uno de cada cuatro jóvenes instalados lo hizo en el marco de un contrato territorial en el periodo analizado. Además de la creación de empleo directo o la instalación de jóvenes, los CTE contribuyeron a elevar la formación de los agricultores (promoviendo la asistencia a cursos de especialización agrícola y ganadera), mejorar las condiciones de trabajo (desde el punto de vista de la higiene y de la seguridad laboral) y regularizar o estabilizar la situación de los asalariados y de las ayudas familiares. 
Figura I. Porcentaje de explotaciones profesionales que han firmado un CTE (por departamentos/provincias)

Más de $15 \%$

De 10 a $15 \%$

De 5 a $10 \%$

Menos de $5 \%$

Fuente: CEMAGREF

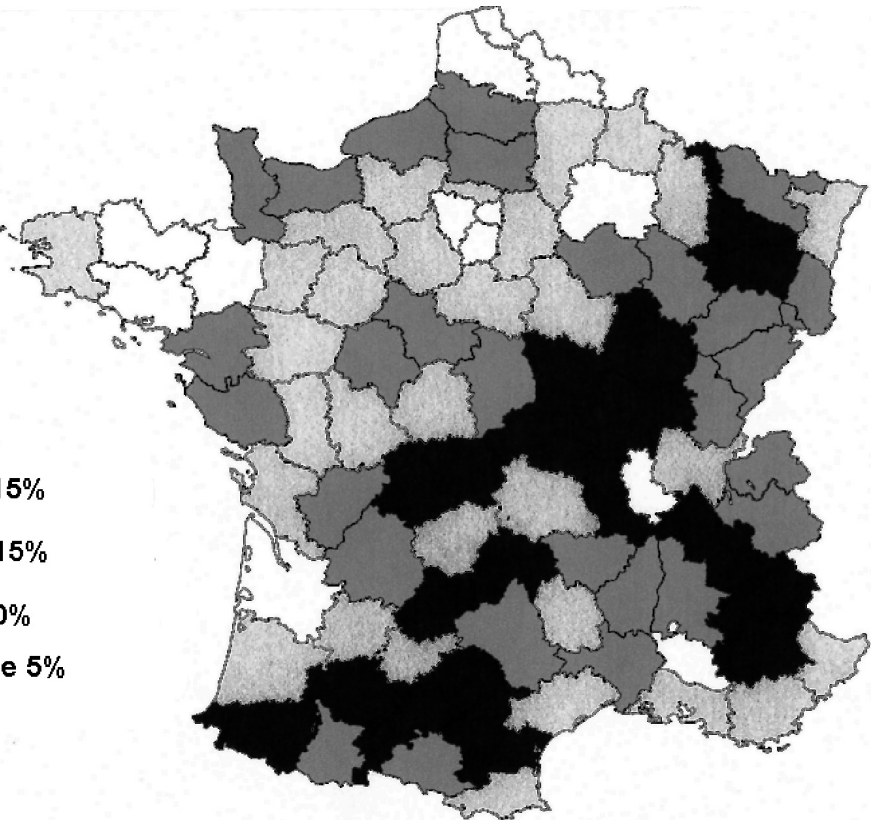

Asimismo, los CTE ayudaron a promocionar la creación de empresas de servicios relacionados con la agricultura, bien para la externalización de diversas labores agrícolas (siembra, recolección, tratamientos fitosanitarios, ...), bien para la realización de tareas de sustitución permitiendo al agricultor disfrutar de periodos de vacaciones o ser ayudado en situaciones de baja por enfermedad. Finalmente, los CTE también contribuyeron a mejorar la gestión de las explotaciones, facilitando la compra de ordenadores y material informático o renovando el parque de maquinaria.

El perfil tipo del signatario de un CTE era el de un agricultor joven (en torno a los 40 años), de buen nivel de formación profesional e instalado en una explotación de superficie algo superior a la media nacional y gestionada mediante la figura jurídica de "sociedad" (Leger, Vollet y Urbano, 2004) (de hecho, el 44\% de los CTE se suscribieron por agricultores integrados en sociedades). Otro dato interesante 
que muestra el potencial innovador de los CTE es el hecho de que uno de cada tres contratos fue firmado por agricultores inscritos en la Reseau d'Agriculture Durable (Red de Agricultura Sostenible).

No todo son laureles en esta experiencia innovadora. Así, el papel desempeñado por la Cámaras Agrarias fue objeto de crítica, centrada en el hecho de que, en algunos departamentos (provincias), los técnicos de estos organismos se dirigían prioritariamente, y como era costumbre, a los agricultores más conocidos, reduciendo así la capacidad de los CTE de alcanzar a aquellos grupos tradicionalmente ignorados por los proyectos modernizadores. En la práctica, los principales signatarios de los CTE en dichos departamentos seguían siendo, por tanto, los agricultores más profesionalizados, con explotaciones de dimensiones económicas por encima de la media e integrados en las dinámicas sectoriales y de filière (Leger, Vollet y Urbano, 2004: 144). La experiencia de estos departamentos muestra cómo un instrumento orientado a la multifuncionalidad y a integrar la dimensión territorial en las estrategias agrarias, necesita para su éxito la complicidad de redes intermedias (los técnicos de los servicios de extensión, pero también los de las organizaciones profesionales) con voluntad de incorporar a nuevos grupos de agricultores. Si eso no ocurre (como fue el caso de los departamentos mencionados), tales instrumentos acaban desnaturalizándose, perdiendo su potencial de innovación y contribuyendo a consolidar el modelo de agricultura que se pretende cambiar.

Otro tipo de críticas que recibieron los CTE en su corto periodo de aplicación estuvo relacionado con la intención de los legisladores (algo ingenua, según los críticos) de hacer realidad la vocación de estos contratos de ser un instrumento para materializar de forma negociada en la agricultura el principio de la multifuncionalidad implicando no sólo a los agricultores, sino también al conjunto de la sociedad local. En este sentido, la experiencia de lo ocurrido en algunos departamentos (provincias) nos dice que no siempre fue así, sino que las organizaciones profesionales agrarias intentaron, y consiguieron, mantener su bloque mayoritario dentro de las comisiones (CDOA), marginando la presencia de otros grupos (como las organizaciones de consumidores o las ambientalistas) y logrando hacer exclusivamente suyo el instrumento de los CTE. Tras cincuenta de años de predominio de un modelo de desarrollo agrario sectorial, basado en la cogestión corporativa entre la agricultura "representada por el sindicalismo mayoritario, es decir, por la FNSEA y el CNJA" y los poderes públicos "encarnados en los organismos agrarios de la administración francesa", la apertura del sector agrario al resto de la sociedad ha demostrado ser un proceso lleno de dificultades y recelos, si no cuenta con el apoyo inicial de los actores mayoritarios o con el apoyo de grupos alternativos dotados de suficiente capacidad de movilización como para neutralizar las inevitables resistencias de 
aquéllos. La realidad nos dice que la resistencia de los sindicatos mayoritarios FNSEA y el CNJA a los CTE no pudo ser contrarrestada ni por el apoyo de los minoritarios MODEF y CNP (llamada esta última a jugar ese papel, pero muy dividida internamente), ni por el de otros grupos sociales (como los consumidores - los ecologistas) dotados de una estructura organizativa demasiado débil como para atender con eficacia al reto de la participación en los debates sobre los CTE desarrollados a nivel de cada departamento (provincia) ${ }^{6}$.

\section{De los CTE a los contratos de agricultura sostenible (CAD)}

Desde que se aprobó la Ley de Orientación Agraria de 1999, la puesta en marcha de los CTE estuvo acompañada de un fuerte debate político-sindical en el que intervinieron con especial virulencia las organizaciones profesionales. La mayoritaria FNSEA recelaba de los CTE como instrumentos de cambio, aceptándolos sólo como vía para compensar la pérdida de renta que se le ocasionaba a los agricultores por realizar una agricultura más sostenible y, por tanto, menos competitiva. Para el CNJA, más interesado por los temas agroambientales, los CTE debían servir para remunerar a todos los agricultores por los servicios que prestan a la sociedad y para incitarlos a practicar una agricultura más respetuosa con el medio ambiente. Por su parte, para la CNP, situada en el flanco izquierdo del panorama sindical agrario francés, los CTE eran valorados por su potencial de cambio y por la función social que debían desempeñar, contribuyendo a una mejor distribución de las ayudas públicas y al mantenimiento del empleo en el medio rural (Moyano y Velasco, 2002). Había, pues, un debate político e ideológico en torno a los CTE, debate que le acompañó en su primera etapa (hasta la derrota de Lionel Jospin en las elecciones presidenciales 2002 y del partido socialista en las legislativas de ese mismo año), y que explica algunos de los cambios acontecidos posteriormente (al acceder el centro derecha al gobierno) y de los que daremos cuenta en este apartado.

A la hora de analizar tales cambios conviene señalar que el disenso de los gobiernos de centro-derecha respecto a los CTE no estaba en la estructura y contenido de

\footnotetext{
${ }^{6}$ En Francia, el tándem formado por la FNSEA y el CNJA ha mantenido la hegemonía desde los años 50 hasta la actualidad (las últimas elecciones a Cámaras Agrarias celebradas en 2007 así lo confirman), seguido de la Coordination Rurale. La CNP es el sindicato más significativo de la izquierda (seguido del MODEF), pero con un nivel de representatividad menor que los otros dos (Moyano, 1988, y Halpin, 2005).
} 
estos contratos, sino que tenía una base política. Sólo por el simple hecho de que habían sido una creación del gobierno socialista en connivencia con el sindicato agrario de izquierda CNP y el movimiento ecologista, los CTE fueron puestos en el ojo del huracán por todas las fuerzas alineadas en el centro y la derecha del espectro político y sindical (la promesa electoral de Chirac en las presidenciales de 2002 de suprimir los CTE, se debía sobre todo a la presión de la FNSEA). Además, el hecho de que parte de su financiación procediera de los fondos obtenidos con la modulación de las ayudas agrícolas del primer pilar de la PAC, fue una baza utilizada por sus críticos para calificar a los CTE de instrumentos politizados y demagogos, ya que, en opinión de la FNSEA, eran vías encubiertas de la izquierda para introducir criterios de justicia social.

Prueba de que la oposición contra los CTE no se dirigía a la existencia misma de una vía contractual para aplicar la nueva política agraria, sino al modo como se financiaba (mediante los fondos del segundo pilar de la PAC y la modulación), es el hecho de que el nuevo gobierno de centro derecha surgido de las elecciones de 2002 no los suprimiera, sino que simplemente les cambiara el nombre, denominándolos CAD (Contrats d'Agriculture Durable) (decreto ${ }^{\circ}$ 2003-675 de 22 de julio de 2003), si bien modificando algunos aspectos de su contenido, estructura y financiación. El CAD mantuvo la naturaleza (heredada de los CTE) de ser un contrato administrativo entre el Estado y el agricultor, manteniéndose también el compromiso de recibir una compensación económica por llevar a cabo durante un periodo de cinco años cambios en la gestión de las explotaciones agrarias en pro de una "agricultura sostenible". La principal diferencia radicaba en los mecanismos de financiación de los $C A D$, que sólo utilizaba los fondos del segundo pilar de la PAC, pero no los procedentes de la modulación de las ayudas del primer pilar de la PAC que tanto revuelo había levantado en el periodo de los CTE.

No obstante, en la cuantía y composición de las ayudas ligadas a los CAD hay diferencias dignas de ser destacadas respecto de los CTE. En efecto, los CAD tienen un límite de ayudas por explotación de 27.000 euros durante los cinco años de duración del contrato, límite que, a diferencia de los CTE, se extiende a los capítulos socioeconómico y medioambiental 7 . El máximo de ayuda que corresponde al capítulo socioeconómico de los CAD es 9.000 euros (frente a los 15.000 euros de los CTE), cifra que representa un tercio de la financiación; los dos tercios restantes

\footnotetext{
${ }^{7}$ Recordemos que los CTE no tenían un máximo designado, sino que estaban formados por dos capítulos presupuestarios: el capítulo socioeconómico, cuya cuantía estaba limitada, y el capítulo medioambiental, que no tenía limitación alguna. En los CAD las dos partidas están limitadas.
} 
(18.000 euros) se destinan a financiar las medidas agroambientales integradas en el capítulo medioambiental de los CAD.

La nueva fórmula de los CAD cambia la función social del contrato, ya que, entre sus objetivos, no está, como sí lo estaba en los CTE, mantener o crear empleo en el medio rural. También cambia la función modernizadora que tenían los CTE, pues en los CAD los planes de mejora de las explotaciones agrarias no se canalizan a través de ellos, sino por vías no contractuales como antaño. De este modo, los $C A D$ se limitan a reproducir los antiguos contratos agroambientales con pequeñas variaciones, incorporando los principios de ecocondicionalidad que desde enero de 2006 están siendo aplicadas en la agricultura a la hora de distribuir las ayudas del primer pilar de la $\mathrm{PAC}^{8}$.

Estas transformaciones no han sido, sin embargo, bien acogidas entre las organizaciones profesionales agrarias, ni siquiera en la propia FNSEA, que había sido la más crítica con los CTE. La disconformidad se explica, en primer lugar, porque la financiación destinada a los CAD se ha reducido casi en un tercio respecto a los $\mathrm{CTE}$; en segundo lugar, porque en la práctica los nuevos contratos restringen la autonomía individual del agricultor (puesto que alguna de las medidas son obligatorias para todo un ámbito territorial) en todo lo que atañe a las decisiones relacionadas con su explotación; y por último en la limitación impuesta al capítulo medioambiental (que en los CTE no tenía límite alguno). Esto ha hecho que las grandes explotaciones agrarias no vean atractivos los $C A D$, y que tampoco los titulares de pequeñas explotaciones encuentren en estos contratos una oportunidad para asegurar su reproducción económica y social, ya que firmar un CAD exige demostrar previamente que la explotación es viable económicamente (exigencia que no existía en los CTE). A esa desconfianza generalizada se suma la obligatoriedad de adjuntar al contrato una declaración de la superficie de la explotación, acompañada de fotografía aérea e incluso de documentaciones contables, exigencia que está en sintonía con el principio de transparencia que ha guiado la última reforma de la PAC (la reforma Fischler de 2003). Todo ello conduce a valorar el reducido impacto de los nuevos CAD a pesar de que se había logrado un elevado consenso entre las organizaciones profesionales agrarias en torno a la conveniencia de continuar con la vía contractual para avanzar en los cambios que necesita la agricultura francesa.

\footnotetext{
${ }^{8}$ A partir de enero de 2006 se ha puesto en funcionamiento el principio de "ecocondicionalidad" de la PAC, es decir el cumplimiento de una serie de normas como condición para obtener las ayudas directas. Junto a dicho principio también se aplicará una modulación de $3 \%$ de las ayudas procedentes del primer pilar para alimentar la financiación del segundo pilar.
} 
Hoy, en efecto, todas las organizaciones profesionales reconocen que los CTE eran instrumentos vanguardistas para incentivar a los agricultores franceses a adaptarse al nuevo escenario de la política agraria y rural, aunque discrepan del momento elegido y de la oportunidad de su instauración. Para la FNSEA, los CTE eran instrumentos que iban demasiado por delante de los cambios acontecidos en la PAC, y cree que el gobierno socialista se precipitó al plantear la financiación de los contratos con fondos de la modulación "entonces, fijada por Bruselas como una decisión no obligatoria para los Estados miembros", rompiendo así la posibilidad de consenso en el sector agrario. Para la CNP, la creación de los CAD es interpretada como una venganza política para desnaturalizar los CTE, rebajando el potencial de cambio de estos contratos al eliminar su dimensión social (excluyendo de sus objetivos la generación de empleo) y dejar prácticamente reducida a la mínima expresión su componente de modernización ecológica y sostenible (sacando de ellos los planes de mejora de las explotaciones). Por su parte, el CNJA, aun compartiendo parte de las críticas esgrimidas por la FNSEA, ha sido más equilibrado en su posición, y puede decirse que, gracias a este sindicato de jóvenes agricultores, la vía contractual se ha mantenido como eje de la política agraria francesa, aunque alejada de los avanzados planteamientos de los CTE. De hecho, lo que ha logrado el CNJA es trasladar a los CAD su planteamiento inicial sobre la necesidad de avanzar en la aplicación de las políticas agroambientales, haciendo de la nueva modalidad de contrato el instrumento idóneo para incitar a los agricultores a desarrollar prácticas agrícolas y ganaderas más respetuosas con el entorno natural.

Esto explica que en los CAD se enfatice, sobre todo, el capítulo de medio ambiente en detrimento del capítulo socioeconómico, siendo criticado precisamente por esto desde determinados círculos de opinión, que ven en estos contratos una reproducción de los contratos agroambientales sin presencia alguna de la dimensión territorial. Como resultado de esas críticas, y quizá para neutralizarlas, el gobierno ha obligado a supeditar las acciones medioambientales de los CAD a las prioridades y especificidades de cada territorio. Paralelamente, se aprobó (en junio de 2005) una Ley para el Desarrollo de los Territorios Rurales, que ha recibido un elevado nivel de consenso y entre cuyos objetivos destacan los siguientes: creación de empleo, rehabilitación de alojamientos en el medio rural, mejora de los servicios públicos en el medio rural y promoción del desarrollo en territorios rurales frágiles y vulnerables. Esta Ley puede verse como resultado de un acuerdo entre los intereses agrarios (sectoriales) y los territoriales (generales), según el cual sale de los CAD la dimensión territorial de la agricultura (concesión a los grupos agraristas) y se regula el territorio mediante una norma general. 
La gradual desnaturalización de los "contratos territoriales" ha culminado con la exclusión de los CDA del nuevo PNDR 2007-2013, donde sólo se mantienen las fórmulas contractuales para la aplicación del programa agroambiental. Paradóïicamente, el impacto de esos contratos, que desbordó las previsiones presupuestarias del periodo 2000-2006, junto a la controversia político-sindical que le acompañó en sus dos etapas, han hecho que, mientras en algunos países de la UE, los "contratos territoriales" estén siendo hoy utilizados como referencia en la aplicación del Reg. 1.698/2005 de desarrollo rural, en Francia se haya retrocedido hacia posiciones anteriores, en una especie de repliegue corporativista donde de nuevo el sindicalismo mayoritario impone sus criterios en materia de política agraria y rural.

\section{Conclusiones}

La creación de los CTE por la Ley francesa de Orientación Agrícola de 1999 fue un modo de anticiparse a la reforma de las políticas agrarias y rurales, respondiendo a los cambios experimentados en la agricultura y el desarrollo rural. El gobierno socialista de L. Jospin tuvo el atrevimiento de poner en marcha nuevos instrumentos de regulación, que, más tarde, serían trasladados a otros países de la UE, como el Reino Unido. En ese sentido podemos calificar a los CTE como instrumentos vanguardistas en la reforma de las políticas públicas dirigidas a la agricultura y el mundo rural.

El carácter vanguardista de los CTE se debía a varios elementos. En primer lugar, al hecho de extender al conjunto de las ayudas agrícolas la vía contractual que hasta entonces sólo se venía utilizando en la aplicación del programa agroambiental, vía que luego estaría en la base de la reforma de la PAC de 2003. En segundo lugar, con los CTE se abría paso un nuevo modelo de desarrollo agrícola, más incluyente e integrador que el anterior modelo de explotaciones especializadas, concretando en la práctica el concepto de multifuncionalidad de la agricultura. En tercer lugar, gracias a los CTE la dimensión territorial toma un lugar preferente a través de un diálogo participativo de los diversos actores implicados en el nivel local, que se concretaba en la asunción de compromisos fijados en función de las necesidades de una zona específica. En cuarto lugar, el verdadero significado de los CTE radicaba en que establecía un nuevo pacto social entre los agricultores y el resto de ciudadanos, según el cual la sociedad reconocía que aquéllos realizan servicios útiles al conjunto de la población y aceptaba remunerarles por ello. En este sentido, cabe decir que la figura de los CTE acerca, por no decir inserta, la política 
agraria en las políticas públicas, introduciendo la idea de que la agricultura es un asunto que compete al conjunto de la sociedad. En quinto lugar, la vía marcada por los CTE avanza en la incorporación de la dimensión medioambiental en la agricultura, tímidamente introducida en el programa agroambiental y reforzada en estos contratos con un capítulo específico dotado de importante financiación. Aparte de eso, quizá lo más innovador de los CTE en materia medioambiental consistiera en concebir las implicaciones ambientales de la agricultura en un sentido amplio y no sólo en sus efectos sobre la contaminación, integrando el medio ambiente y el territorio a través de aspectos como el paisaje, el patrimonio natural y cultural, el uso del agua y el suelo. Con ese planteamiento, se incorporaban el medio ambiente y el territorio como unas dimensiones más de un nuevo perfil profesional de los agricultores (menos especializado y más polivalente). En sexto lugar, por último, el aspecto participativo de los CTE recogía la filosofía ascendente (bottom-up) de los programas Leader anticipándose así a lo que más tarde hará la Comisión Europea al integrar esta metodología en el Reg. 1.698/2005 de desarrollo rural.

La experiencia de los CTE permite preguntarse si tras estos contratos había o no voluntad política de contrarrestar las desigualdades sociales generadas por la modernización productivista frenando la desaparición de aquellas formas sociales de producción no competitivas, reflexión que se puede trasladar hoy al debate sobre la multifuncionalidad de la agricultura (Gómez-Limón y Barreiro, 2007) e incluso a las nuevas orientaciones de la política agraria europea (como el desacoplamiento de las ayudas agrícolas). En esta nueva forma de gestionar la agricultura se inscriben derechos y deberes, compromisos o contratos, que regulan la distribución de rentas entre los agricultores. Se produce, en definitiva, una ordenación de las distintas categorías de agricultores, estableciendo prioridades y diferenciando los instrumentos de gestión: de un lado, políticas destinadas a los agricultores con explotaciones viables que no precisan ayudas directas, pero sí incentivos para mejorar su eficiencia y competitividad; $y$, de otro, políticas destinadas a garantizar las rentas de los agricultores con explotaciones difícilmente viables en mercados abiertos, pero a los que se les ofrece la posibilidad de diversificar sus rentas y asegurar su reproducción económica, ya que la utilidad social de este grupo no será valorada ya sólo por la producción, sino por la prestación de servicios a la comunidad. 


\section{Referencias bibliográficas}

AlPHANDERY, P. (2001), Les campagnes françaises de l'agriculture á l'énvironnement (1945-2000). Politiques publiques, dynamiques sociales et enjeux territoriaux, Tesis doctoral, Paris, Institut d'Etudes Politiques.

BAZIN, G. y Roux, B. (1995), "Resistence to marginalisation in Mediterranéen rural regions", Sociologia Ruralis, Vol. XXXV, pp. 335-347, Assen, ESRS.

Buller, H. (1996), "Regards croisés, Anglaterre, Irlande, France", Études Rurales, $n^{\circ} 141-142$.

BUTTEL, F. (1993), "Environmentalization and greening: origins, processes and implications", en S. HARPER (ed.), The greening of rural policy: international perspectives, Londres y Nueva York, Belhaven Press, pp. 12-26.

CANTÓ LóPEZ, Ma Teresa (2004), "La protección voluntaria del ambiente agrario: de la subvención al contrato territorial de explotación", en E. ARGULLOL MURGADAS (coord.), La dimensión ambiental del territorio frente a los derechos patrimoniales: un reto para la protección efectiva del medio natural, pp. 409-425.

Coulomb, P. y NaLLet, H. (1980), Le syndicalisme agricole et la création du paysan modèle, París, INRA-CORDES.

Delgado, Ma del M. (2004), La política rural europea en la encrucijada, Madrid, Servicio de Publicaciones del Ministerio de Agricultura, Pesca y Alimentación (Serie Estudios).

- (2005), "La política rural europea: de Cork a Salzburgo", en E. Moyano (coord.), XI Informe de la Agricultura Familiar en España, Madrid, Fundación de Estudios Rurales.

GARRIDO-FeRnÁNDEZ, F. (2000), La cuestión ambiental en la agricultura, Málaga, Unicaja.

- (2006), "Los agricultores como actores de la política agroambiental. Un enfoque multidimensional", Papers. Revista de Sociología, n 81, pp. 37-62.

Gómez-Limón, J. A. y BARreiro, J. (coords.) (2007), La multifuncionalidad de la agricultura, Madrid, Eudema. 
GRUPO DE BRUGGE-BRUJAS (1997), Por un cambio necesario en la agricultura europea, Madrid, Servicio de Publicaciones del MAPA.

HalpIN, D. (ed.) (2005), Surviving Global Change? Agricultural Interest Groups in Comparative Perspective, Aldershot, Ashgate.

Hervieu, B. (1996), Los campos del futuro, Madrid, Servicio de Publicaciones del MAPA, Serie Estudios.

LEGER, F.; Vollet, D. y URBANo, G. (2004), "Le CTE: un bilan en di-teintes révélateur des tensions de l'agriculture française?", Les cahiers de la multifonctionalité, $n^{\circ} 7$.

MoYANO, E. (1988), Sindicalismo y Política Agraria en Europa. Los casos de Francia, Italia y Portugal, Madrid, Servicio de Publicaciones del MAPA, Serie Estudios.

- (2005), "Nuevas orientaciones de la política europea de desarrollo rural", Revista de Fomento Social, vol. 60, n² 238, abril-junio, pp. 219-241.

Moyano, E. y Velasco, A. (2002), Los Contratos Territoriales de Explotación (CTE). Un instrumento de cambio en la política agraria francesa, Córdoba-Sevilla, IESA-CSIC y Consejería de Agricultura de la Junta de Andalucía.

Paniagua, A. (coord.) (2001), Naturaleza, agricultura y política ambiental, Colección Politeya, CSIC, Madrid.

ReMY, J. (2001), "La co-institution des contrats territoriaux d'exploitation", Ingénieries (n monográfico), vol. 2, pp. 45-54.

- (2004), "La profesionalización de los agricultores en Francia", XI Informe Socioeconómico de la Agricultura Española, Madrid, Fundación de Estudios Rurales, pp.114-135.

Ramos, E.; Romero, J. J.; Romero, M. y Ambrosio, M. (2001), "Hacia un contrato territorial de explotación", en E. RAMOs (coord.), El desarrollo rural en la Agenda 2000, Madrid, Servicio de Publicaciones del Ministerio de Agricultura, Pesca y Alimentación (Serie Estudios).

SERVOLIN, C. (1989), Las políticas agrarias, Madrid, Servicio de Publicaciones del Ministerio de Agricultura, Pesca y Alimentación (Serie Estudios). 
VeLASCO, A. (2002), "Los contratos territoriales y la reproducción de las pequeñas explotaciones agrarias", IX Informe Socioeconómico de la Agricultura Española, Madrid, Fundación de Estudios Rurales, pp. 126-132.

WнІтВY, M. (1997), The European Environment and the CAP Reform, Wallingford, $C A B$ International. 